\title{
Exosomes of stem cells from human exfoliated deciduous teeth as an anti- inflammatory agent in temporomandibular joint chondrocytes via miR-100-5p/mTOR
}

\author{
Ping Luo ${ }^{1,2,3+}$, Chao Jiang ${ }^{1,2,3+}$, Ping $\mathrm{Ji}^{1,2,3}$, Menghong Wang ${ }^{1,2,3,5^{*}}$ and Jie $\mathrm{Xu}^{1,2,3,4^{*}}$ CD
}

\begin{abstract}
Objectives: Temporomandibular joint osteoarthritis (TMJOA) is an inflammatory joint disease. This study investigated whether exosomes (ExOS) of stem cells from human exfoliated deciduous teeth (SHEDs) have a therapeutic effect on TMJ inflammation and elucidated the underlying mechanisms.
\end{abstract}

Materials and methods: SHEDs were verified by flow cytometry. SHED-Exos were identified by western blotting, nanoparticle tracking analysis, and transmission electron microscopy. Western blot and RT-qPCR were performed to verify the anti-inflammatory effects of SHED-Exos. MicroRNA (miRNA) array analysis was conducted to determine the miRNA expression profiles of SHED-EXos, and the key pathways were analyzed. After chondrocytes were treated with an miR-100-5p mimic or rapamycin, relative expression of genes was measured by RT-qPCR and western blotting. A luciferase reporter assay was performed to reveal the molecular role of the exosomal miR-100 target, mTOR.

Results: MiR-100-5p was enriched in the SHED-Exos. Treatment with SHED-Exos suppressed the expression of interleukin-6 (IL-6), IL-8, matrix metalloproteinase 1 (MMP1), MMP3, MMP9, MMP13, and disintegrin and metalloproteinase with thrombospondin motifs 5 (ADAMTS5). Chondrocytes treated with the miR-100 mimic showed lower expression of MMP1, MMP9, MMP13, ADAMTS5, and mTOR. In contrast, miR-100 downregulation upregulated the MMPs and mTOR. Rapamycin treatment upregulated miR-100 and downregulated MMPs and ADAMTS5. Furthermore, the luciferase reporter assay demonstrated that miR-100-5p directly targeted the mTOR 3' untranslated region and that SHED-Exos miR100-5p repressed mTOR expression.

Conclusions: This study demonstrated that SHED-EXos suppress inflammation in TMJ chondrocytes and may thus be a novel therapeutic agent for TMJ inflammation.

Keywords: Osteoarthritis, SHED, Exosomal microRNA, Inflammation, Temporomandibular joint

\section{Introduction}

The temporomandibular joint (TMJ) is a synovial joint that performs the most complicated movement in the human body. Osteoarthritis (OA) is a degenerative disease characterized by progressive cartilage degradation, subchondral bone remodeling, synovitis, and chronic pain [1]. Recent studies showed that inflammation is strongly involved in the pathogenesis of TMJOA, which leads to high

\footnotetext{
*Correspondence: wmh@hospital.cqmu.edu.cn; xujie@hospital.cqmu.edu.cn ${ }^{\dagger}$ Ping Luo and Chao Jiang contributed equally to this work.

${ }^{1}$ College of Stomatology, Chongqing Medical University, Chongqing, China Full list of author information is available at the end of the article
}

expression of inflammatory cytokines, including interleukin-6 (IL-6) and IL-8 [2]. Increased expression of inflammatory factors causes pathological changes in chondrocytes, thereby upregulating catabolic enzymes in the cartilage matrix. Excess production of matrix-degrading proteases including matrix metalloproteinases (MMPs) and a disintegrin and metalloproteinase with thrombospondin motifs (ADAMTSs), results from disruption of metabolic homeostasis and leads to cartilage degradation with inflammation $[3,4]$. IL-1 $\beta$, a major proinflammatory cytokine, is implicated in the destructive effects characterized by increasing cartilage degradation and suppression of cartilage matrix

(c) The Author(s). 2019 Open Access This article is distributed under the terms of the Creative Commons Attribution 4.0 International License (http://creativecommons.org/licenses/by/4.0/), which permits unrestricted use, distribution, and 
synthesis [5, 6]. There are several current treatment strategies for relieving inflammation and preventing the degradation of the joint complex. However, no effective strategy exists to repair and regenerate the damaged TMJ.

Lately, there has been an increasing interest in the use of mesenchymal stem cells (MSCs) for the treatment of TMJOA [7]. Stem cells from human exfoliated deciduous teeth (SHEDs) are an ideal cell source for regenerative medicine as they are highly proliferative, have high multipotency, and are immunosuppressive [8-11]. It is well-documented that MSCs release a rich secretome containing large amounts of cytokines, growth factors, chemokines, and extracellular nanoparticles. The most important nanoparticles shed by MSCs are exosomes [12, 13]. Recently, increasing evidence has demonstrated that the small membrane vesicles called exosomes play a key role in the mechanism of action in stem cell therapy [12, 14]. Exosomes are small membrane-bound vesicles (30-100 $\mathrm{nm}$ in diameter) that are released into the extracellular space by most cell types under both physiological and pathological conditions $[15,16]$. Exosomes contain microRNAs (miRNAs), mRNAs, DNA, and signaling proteins and can modulate the activities of recipient cells [12]. Exosomes have been identified as the principal agent mediating the therapeutic efficacy of SHEDs in several medical conditions, such as traumatic brain injury [17], wound healing [18], and acute inflammation [19]. Moreover, it is believed that exosomes hold great promise as a cell-free therapy because of their safety and low immunogenicity [20].

Exosome-borne miRNAs have been proposed as a means of intercellular communication. MiRNAs are small 18-24 nucleotide-long noncoding RNAs that control gene expression post-transcriptionally [21]. MiRNAs play a key part in cellular proliferation, development, and tissue remodeling [22]. However, it is still unknown which miRNAs are expressed in SHED exosomes (SHED-Exos) and how they are regulated.

Therefore, we hypothesized that SHED-Exos could be beneficial and serve as a promising treatment for TMJOA. In this study, we aimed to explore the therapeutic potential of SHED-Exos and their mechanisms of action in TMJOA. This study may thus help develop a prophylactic agent for TMJOA.

\section{Materials and methods}

\section{Ethics statement for human samples}

Ethics approval was obtained from the Committee of Chongqing Medical University, and written informed consent was obtained from all the participants. Collection and processing of condylar and human exfoliated deciduous teeth was conducted in accordance with the Declaration of Helsinki.

\section{Cell culture}

SHEDs were purchased from China Oral Stem Cell Bank (Beijing, China) and originated from nine normal human deciduous incisors collected from 5- to 8-year-old individuals. SHEDs were plated in T175 culture flasks in $\alpha$-MEM supplemented with $10 \%$ fetal bovine serum (FBS) and were incubated at $37{ }^{\circ} \mathrm{C}$ in an atmosphere containing $5 \%$ $\mathrm{CO}_{2}$ at $100 \%$ humidity. To obtain chondrocytes, human condyles were collected from five patients with condylar fracture during the surgical procedure of mandibular condylectomy. Cartilage were immediately isolated from the TMJ cartilage layer with a surgical blade, and carefulness was taken not to disturb the subcondral bone. All cartilage specimens were washed three times with phosphate-buffered saline (PBS) to remove blood prior to mincing and digestion for $30 \mathrm{~min}$ in $0.25 \%$ trypsin (HyClone, USA) followed by digestion with $3 \mathrm{mg} / \mathrm{ml}$ type I collagenase for $1 \mathrm{~h}$. The chondrocytes were washed, resuspended in DMEM (HyClone, USA) supplemented with 10\% FBS (HyClone, USA), 1\% penicillin/streptomycin solution, and cultured at $37^{\circ} \mathrm{C}$ and $5 \% \mathrm{CO}_{2}$. The chondrocytes were then cultured in dishes at a density of $1 \times 10^{5} \mathrm{cells} / \mathrm{cm}^{2}$. The cells used in the experiments were at passage 4 . For gene and protein expression experiments, chondrocytes were seeded at $1 \times 10^{5} \mathrm{cells} / \mathrm{cm}^{2}$ in a 6 -well culture plate. The chondrocytes were then pretreated with SHED conditioned medium (SHED-CM) or SHED-Exos $(150 \mu \mathrm{l}$ per well, equivalent to exosomes from $1.5 \times 10^{7}$ cells) for $2 \mathrm{~h}$ followed by stimulation with the proinflammatory factor $\mathrm{IL}-1 \beta(10 \mathrm{ng} / \mathrm{ml})$. For gene expression analysis, samples were harvested after $24 \mathrm{~h}$, whereas for protein expression analysis, samples were harvested at $48 \mathrm{~h}$.

\section{Isolation of exosomes}

SHEDs were seeded and grown to confluence in a T175 culture flask. Exosomes were isolated from the culture medium of cells containing exosome-free FBS. One day prior to isolation, the cells were washed with serum-free medium and cultured for $48 \mathrm{~h}$. Exosomes were isolated in accordance with previously published protocols [23]. The exosome purification procedure was based on differential ultracentrifugation. All centrifugation steps were performed at $4^{\circ} \mathrm{C}$. First, to eliminate dead cells and large cell debris, culture supernatants collected from SHEDs were centrifuged successively at increasing speeds $(300 \times g$ for 10 $\mathrm{min}, 2000 \times g$ for $10 \mathrm{~min}$, and $20,000 \times g$ for $30 \mathrm{~min})$. The resultant supernatants were ultracentrifuged at $100,000 \times \mathrm{g}$ for 70 min in an ultracentrifuge (Hitachi, CP100NX, Japan). Finally, the pellets were washed with $10 \mathrm{ml}$ of PBS and centrifuged one last time at $100,000 \times g$ for $70 \mathrm{~min}$ to eliminate contaminating proteins. Final pellets of the exosomal fraction were resuspended in sterile $100 \mu \mathrm{l}$ PBS and stored at $-80^{\circ} \mathrm{C}$. Exosome suspensions were normalized to the cell 
number and diluted to ensure that $100 \mu \mathrm{l}$ of suspension contained exosomes isolated from $1 \times 10^{7}$ cells.

\section{SHED-CM}

The conditioned medium (CM) from SHEDs was generated as follows: SHEDs (passage 3-5) were seeded in a T175 cell culture flask for $24 \mathrm{~h}$ at $37^{\circ} \mathrm{C}$ with $5 \% \mathrm{CO}_{2}$. Next, the culture medium was removed from each flask, and the flasks were washed twice with PBS to remove cell debris. The SHEDs were then cultured in serum-free medium for $48 \mathrm{~h}$. The SHED-CM was collected and centrifuged at $1500 \mathrm{rpm}$ for $5 \mathrm{~min}$, followed by centrifugation at $3000 \mathrm{rpm}$ for $5 \mathrm{~min}$ to remove cell debris.

\section{Identification of SHEDs}

SHEDs (passage 3) were verified using flow cytometry to assess the phenotypic characteristics. CD73 ((BD, USA), CD146 ((BD, USA), and CD34 ((BD, USA) were used for detection. To identify the multiple differentiation potential of SHEDs, cells were induced to differentiate in osteogenic and adipogenic medium for 3 weeks. Flow cytometry and differentiation protocols for SHEDs were performed as described previously [24]. Alizarin Red (Solarbio, China) and Oil Red O (Solarbio, China) were used to identify these cell types, respectively.

\section{Transmission electron microscopy (TEM)}

TEM was performed to verify the presence of exosomes in purified samples. The enriched exosomes were placed onto formvar/carbon-coated nickel TEM grids and incubated for $30 \mathrm{~min}$. With the excess fluid removed, the samples were then stained with $3 \%$ phosphotungstic acid (pH 6.9) for $5 \mathrm{~min}$ and then subjected to TEM.

\section{Nanoparticle tracking analysis (NTA)}

A Nanosight Tracking Analysis system (Brookhaven Instruments Corp, USA) was employed to determine the particle size and particle concentration per milliliter.

\section{Fluorescent labeling of exosomes: endocytosis experiments}

The exosomes were labeled with the PKH-67 Labeling Kit (Sigma, USA) according to the manufacturer's protocol. A control reaction was performed with PBS instead of exosomes during each labeling reaction, which served as a control in subsequent experiments to account for nonspecific staining resulting from the labeling procedure. SHEDs were seeded in 6-well culture plates. At $24 \mathrm{~h}$ after cell seeding, the labeled exosomes or a control mixture were added to the culture medium and incubated for 30 min, $1 \mathrm{~h}$, or $2 \mathrm{~h}$ at $37^{\circ} \mathrm{C}$. The coverslips were washed thrice with PBS and fixed in 4\% neutral buffered formalin solution, and the nuclei were stained with 4',6-diamidine2 '-phenylindole (DAPI) according to previously published protocols [25]. Then, images were captured using an Olympus microscope (Olympus, Japan).

\section{Firefly luciferase and Renilla luciferase assays}

293T cells were seeded in a 96-well plate, cultured to $70 \%$ confluence, and transfected with either the h-mTOR-3' UTR plasmid or hsa-miR-100-5p/Negative Control (Hanbio Biotechnology, China) using a transfection reagent (Hanbio Biotechnology, China). The cells were collected at $48 \mathrm{~h}$ after transfection. Luciferase activity was determined in cell lysates with a Dual-Luciferase Reporter Assay System (Promega, USA) according to the manufacturer's instructions. Firefly luciferase and Renilla luciferase activities were detected on a Veritas Microplate Luminometer (Promega, USA). The firefly luciferase to Renilla luciferase ratio was calculated for each sample and was normalized to the ratio for NG-cultured cells.

\section{Reverse transcription-quantitative PCR (RT-qPCR) for mRNA and miRNA expression analyses}

For gene expression analysis, cells were collected, washed thrice with PBS, and lysed in RNAiso plus (Takara, Japan). Total RNA was isolated and reverse-transcribed using the PrimeScript ${ }^{\text {ti }}$ RT Reagent Kit with gDNA Eraser (Takara, Japan) to obtain cDNA. For miR-100 expression analysis, after RNA isolation, first-strand cDNA was synthesized via reverse transcription using the Mqklir- $\mathrm{X}^{\mathrm{Tw}}$ miRNA First Strand Synthesis Kit (Takara, Japan) according to the manufacturer's instructions. qPCR was then carried out on a Bio-Rad real-time PCR system (CFXConnect, USA) for 40 cycles with the Power TB Green PCR Master Mix (Takara, Japan). The expression levels of target genes were normalized to the control housekeeping gene GAPDH. MiR-100 expression was normalized to that of $U 6$. Gene expression data were analyzed by the $2^{-\Delta \Delta \mathrm{Ct}}$ method. For primer sequences, refer to Table 1.

\section{Western blotting}

Chondrocytes were pretreated with SHED-Exos or SHED-CM and then incubated with or without $10 \mathrm{ng} /$ $\mathrm{mL}$ IL-1 $\beta$ for $48 \mathrm{~h}$ before the cell lysates were prepared. For preparation of SHED-Exos, a purified exosome pellet was resuspended in RIPA Lysis Buffer (Beyotime, China). To prepare total-cell lysates, cells were washed twice with cold PBS and lysed in RIPA Lysis Buffer (Beyotime, China) for $15 \mathrm{~min}$ on ice. Next, the exosomes were pelleted and total protein concentration was quantified using the Enhanced BCA Protein Assay Kit (Beyotime, China). For western blot analysis, $30 \mu \mathrm{g}$ of cell lysates or SHED-Exo lysates were loaded for each sample and separated by $10 \%$ SDS-PAGE. The proteins were transferred to polyvinylidene difluoride membranes and then blotted with antibodies against calnexin (1:1000, CST, USA), CD9 (1:800, ProteinTech, USA), CD63 (1:1000, Abcam, 
Table 1 Primer sequences for RT-qPCR

\begin{tabular}{|c|c|c|}
\hline Gene & Forward primer $\left(5^{\prime}-3^{\prime}\right)$ & Reverse primer $\left(5^{\prime}-3^{\prime}\right)$ \\
\hline IL-6 & AGCCCACCGGGAACGA & GGACCGAAGGCGCTTGT \\
\hline $\mid L-8$ & AGAAGTTITGAAGAGGGCTGAGA & AGTTTCACTGGCATCTTCACTGATT \\
\hline MMP1 & ACTGCCAAATGGGCTTGAAG & TTCCCTTGGAAAACCGGACTT \\
\hline MMP3 & GAGGCATCCACACCCTAGGTT & TCAGAAATGGCTGCATCGATT \\
\hline MMP9 & CCCTTGTGCTCTTCCCTGGA & TCTGCCACCCGAGTGTAACC \\
\hline MMP13 & ATTAAGGAGCATGGCGACTTCT & CCCAGGAGGAAAAGCATGAG \\
\hline ADAMTS5 & GGCCTCCATCGCCAATAGG & GGATAGCTGCATCGTAGTGCT \\
\hline miR-100 & GTGTTCAAGCCTAGATGCCCAA & GCATCTAGGCTTGAACACGCC \\
\hline mTOR & TCTTTCATTGGAGACGGTTTGG & AGGTITCATGGGATGTCGCT \\
\hline U6 & CGCTTCGGCAGCACATATAC & AAATATGGAACGCTTCACGA \\
\hline GAPDH & CTITGGTATCGTGGAAGGACTC & GTAGAGGCAGGGATGATGTTCT \\
\hline
\end{tabular}

IL-6 Interleukin-6, IL-8 Interleukin-8, MMP1 matrix metalloproteinase-1, MMP3 matrix metalloproteinase-3, MMP-9 matrix metalloproteinase-9, MMP13 matrix metalloproteinase-13, ADAMTS5 disintegrin and metalloproteinase with thrombospondin motifs 5, miR-100 microRNA-100, mTOR mammalian target of rapamycin, $G A P D H$ glyceraldehyde 3 phosphate dehydrogenase, $R T$-qPCR Reverse transcription-quantitative polymerase chain reaction

USA), TSG101 (1:800, ProteinTech, USA), MMP1 (1:1000, Bioss, China), MMP9 (1:800, Zen Bioscience, China), and mTOR (1:500, CST, USA). GAPDH (1:2000, Bioss, China) was blotted as the loading control. Images were captured and analyzed with a computer program (ImageJ, USA). The presented blots are representative of three separate reproducible experiments.

\section{Bioinformatics analysis}

The sequences that matched the human genome were chosen for subsequent analysis. miRNA identification was performed in the miRBase database, and the miRNA expression levels were normalized to transcripts per million clean tags. Microarray analyses were performed on the miRNA using an Affymetrix GeneChip miRNA 4.0 Array at the Integrated Genomics Core, Augusta University, GA. Kyoto Encyclopedia of Genes and Genomes (KEGG) pathway analysis was then performed in KOBAS3.0 (http:// kobas.cbi.pku.edu.cn/) to determine the participation of co-expressed genes in different biological pathways. TargetScan (http://www.targetscan.org) and miRanda (http:// www.microrna.org) were employed to determine the potential miR-100-5p-binding site.

\section{Transfection of miRNA-100}

Chondrocytes were seeded on 6-well culture slides and grown to $70 \%$ confluence. The cells were transfected with $50 \mathrm{nM}$ miR-100 mimic, $100 \mathrm{nM}$ miR-100 inhibitor, or the corresponding control oligonucleotide (miR-100-mimic-NC or miR-100-inhibitor-NC) using the GP-siRNA-mate-plus transfection reagent (GenePharma, China) in $2 \mathrm{ml}$ of $\alpha$ MEM according to the manufacturer's instructions. Six $h$ post-incubation, the medium was removed, and cells were washed thrice with PBS and transfected for $48 \mathrm{~h}$ in standard conditions. The sequences were as follows: hsa-miR-100-5p mimic (sense, 5'-AACCCGUAGAUCCGAACUUGUG-3'; antisense, 5'-CACAAGUUCGGAUCUACGGGUU-3'), hsamiR-100-5p inhibitor (sense, 5'-CACAAGUUCGGAUCUA CGGGUU-3'), mimic negative control (sense, $5^{\prime}$-UUUGUA CUACACAAAAGUACUG-3'; antisense, 5'-CAGUACUU UUGUGUAGUACAAA-3'), and inhibitor negative control (sense, 5'-CAGUACUUUUGUGUAGUACAAA-3'). All the oligos were synthesized by RiboBio (Guangzhou, China).

\section{Treatment with an mTOR inhibitor}

Chondrocytes were incubated with an mTOR inhibitor: rapamycin $(10 \mathrm{nM})$ (Selleck, USA), for $24 \mathrm{~h}$, and then stimulated with IL-1 $\beta$ for $24 \mathrm{~h}$ followed by mRNA expression analysis.

\section{Statistical analysis}

All procedures were repeated at three biological replicates to verify the results. GraphPad Prism 7.0 (GraphPad Software, USA) was employed to perform statistical analysis. Data are presented as mean \pm SEM. Results were statistically analyzed with Student's $t$ test for two-group comparisons and using one-way ANOVA with Tukey's post hoc test for multigroup comparisons. Ns indicates no significant difference. Data with $P$ values $<0.05$ were considered significant. $P<0.05$, $P<0.01, P<0.001$, and $P<0.0001$ in figures indicate statistically significant differences and are represented as $*, \cdots,{ }^{*}, * *$, and ${ }^{* * * * * *}$, respectively.

\section{Results}

\section{Characterization of SHED and their differentiation potential}

SHEDs were characterized by flow cytometry after staining surface markers. These cells were found to be positive for CD73 and CD146, but did not express the hematopoietic marker CD34 (Fig. 1a). Trilineage differentiation potential was evaluated by staining with Alizarin red for osteogenic (Fig. 1b) and Oil red O for adipocytic (Fig. 1c). 


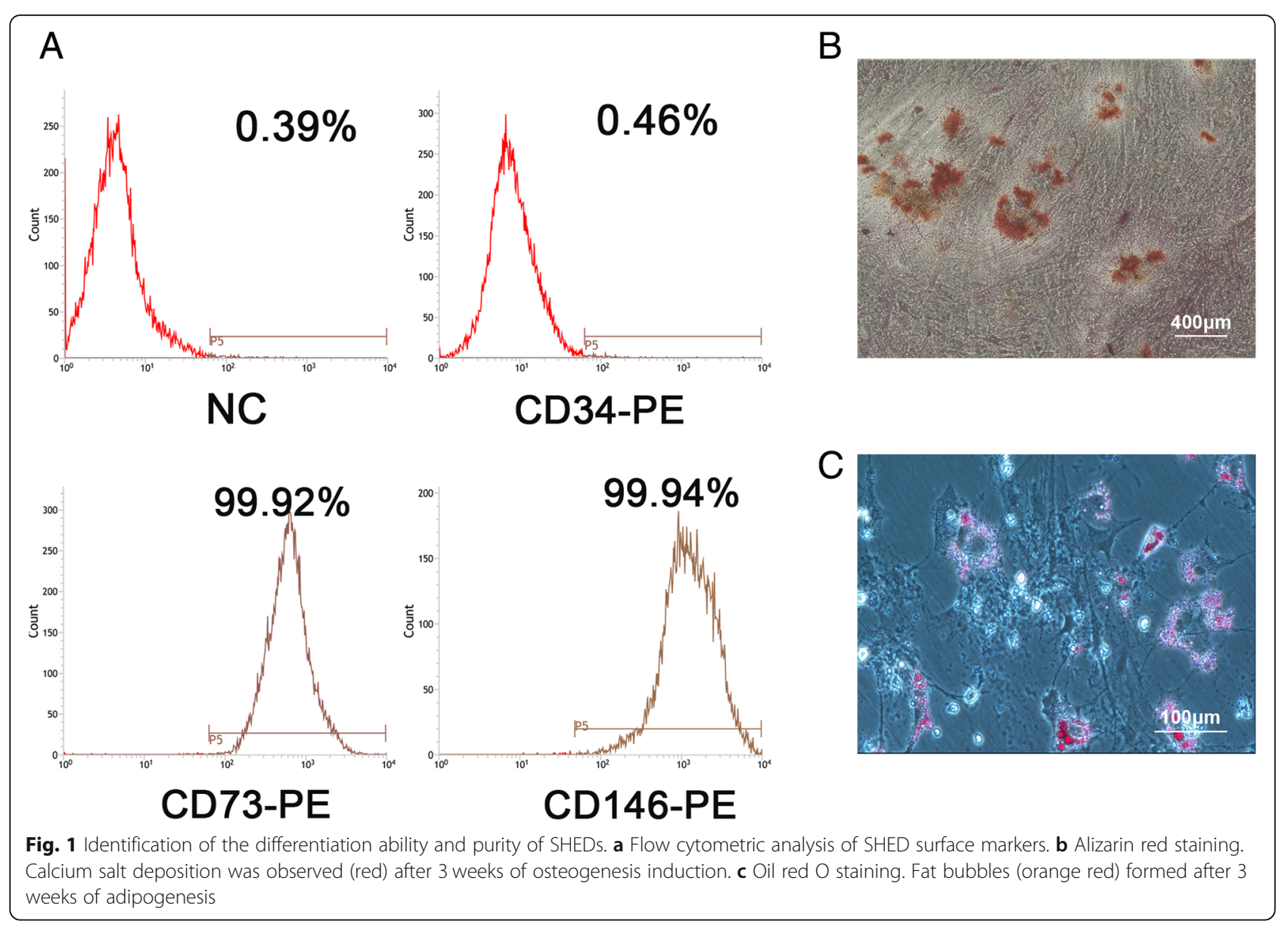

\section{SHED-Exo identification}

To verify the presence of SHED-Exos in the isolates, TEM, western blotting, and NTA were performed. The electron micrographs showed that most of the exosomes had a characteristic morphology with sizes ranging from 30 to $100 \mathrm{~nm}$ and appeared as circular particles (Fig. 2a). Then, the expression of surface exosomal markers CD9, CD63, and TSG101 was verified by western blotting. The results indicated that SHED-Exos carried large amounts of CD9, CD63, and TSG101 compared to SHED lysates. Besides, the data showed no expression of the endoplasmic reticulum chaperone protein calnexin in SHED-Exos, suggesting that the exosomes extracted from the conditioned medium $(\mathrm{CM})$ contained no cellular components. (Fig. 2b). NTA revealed that the size distribution of Exos was near $100 \mathrm{~nm}$, in agreement with the characteristics of exosomes (30-100 nm; Fig. 2c). Overall, we successfully obtained exosome particles from SHEDs.

\section{SHED-Exos suppress proinflammatory cytokine expression} in chondrocytes

To investigate the anti-inflammatory effects of SHEDExos, chondrocytes were pretreated with SHED-Exos or SHED-CM for $2 \mathrm{~h}$, followed by IL- $1 \beta$ stimulation. RT-
qPCR analyses revealed that the expression levels of proinflammatory cytokines IL- 6 and IL-8, and the proteases MMP1, MMP3, MMP9, MMP13, and ADAMTS5 were suppressed in the SHED-Exo-treated group and SHEDCM-treated group (Fig. 3a). Further, the results of protein analyses were consistent with these results, i.e., MMP1, MMP9, and MMP13 were downregulated in the SHEDExo-treated cells and SHED-CM-treated cells (Fig. 3b, c). To examine whether the exosomes released by SHED were translocated into chondrocytes, endocytosis of exosomes by SHEDs was tested. The cultures were incubated with labeled exosomes at $37^{\circ} \mathrm{C}$ for $30 \mathrm{~min}, 1 \mathrm{~h}$, and $2 \mathrm{~h}$. The isolated exosomes were labeled fluorescently (green), and chondrocyte nuclei were stained blue. Figure $3 \mathrm{~d}$ reveals that the SHED-Exos were endocytosed by chondrocytes at $30 \mathrm{~min}, 1 \mathrm{~h}$, and $2 \mathrm{~h}$ and that the green fluorescence increased with incubation time.

\section{SHED-Exos ameliorate inflammation through miR-100}

The expression profile of miRNAs in SHED-Exos was determined by miRNA microarray analysis. The results uncovered the top 20 most significant differentially expressed exosomal miRNAs. MiR-100 was more abundant in SHED-Exos (Fig. 4a). To determine whether exosomal 

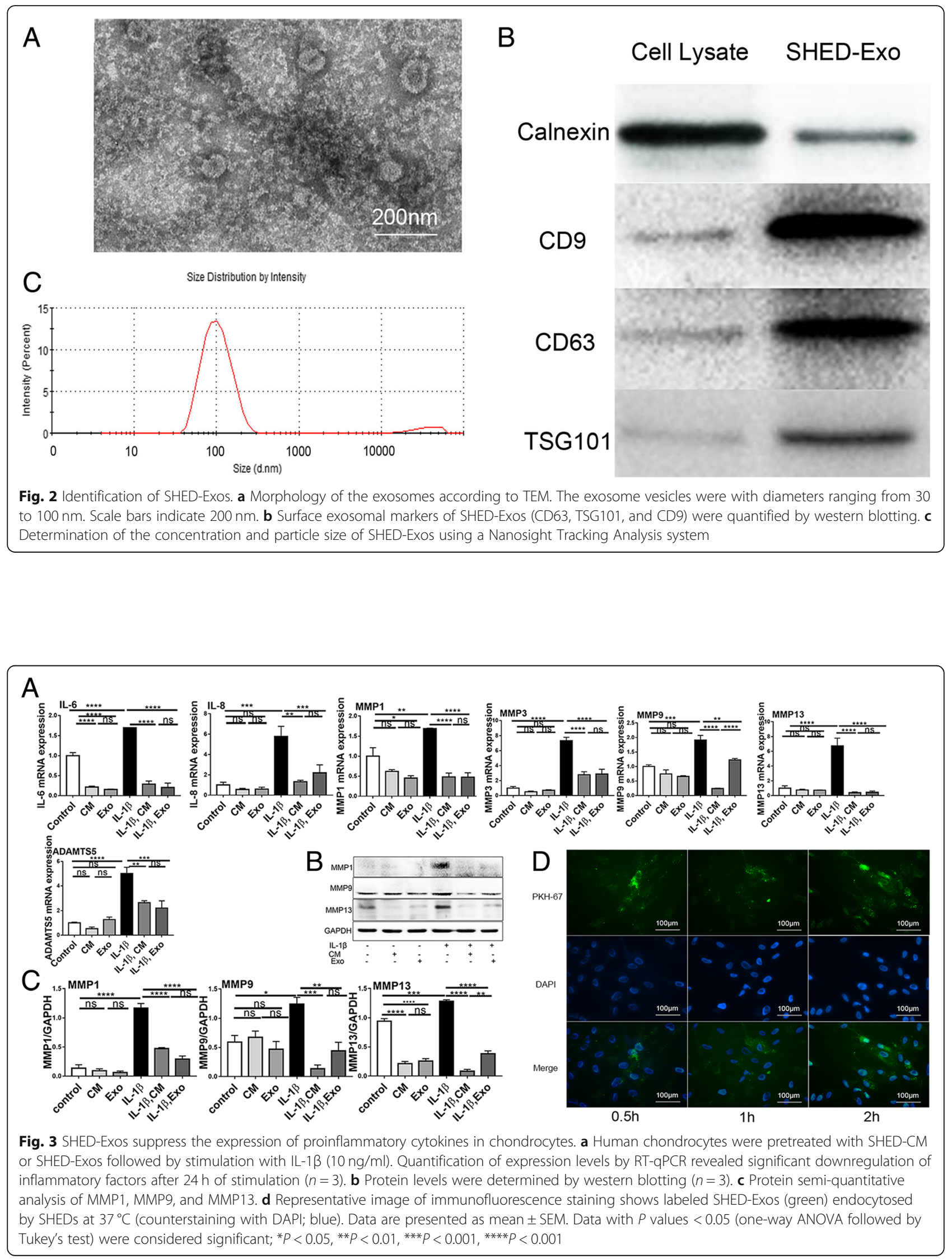


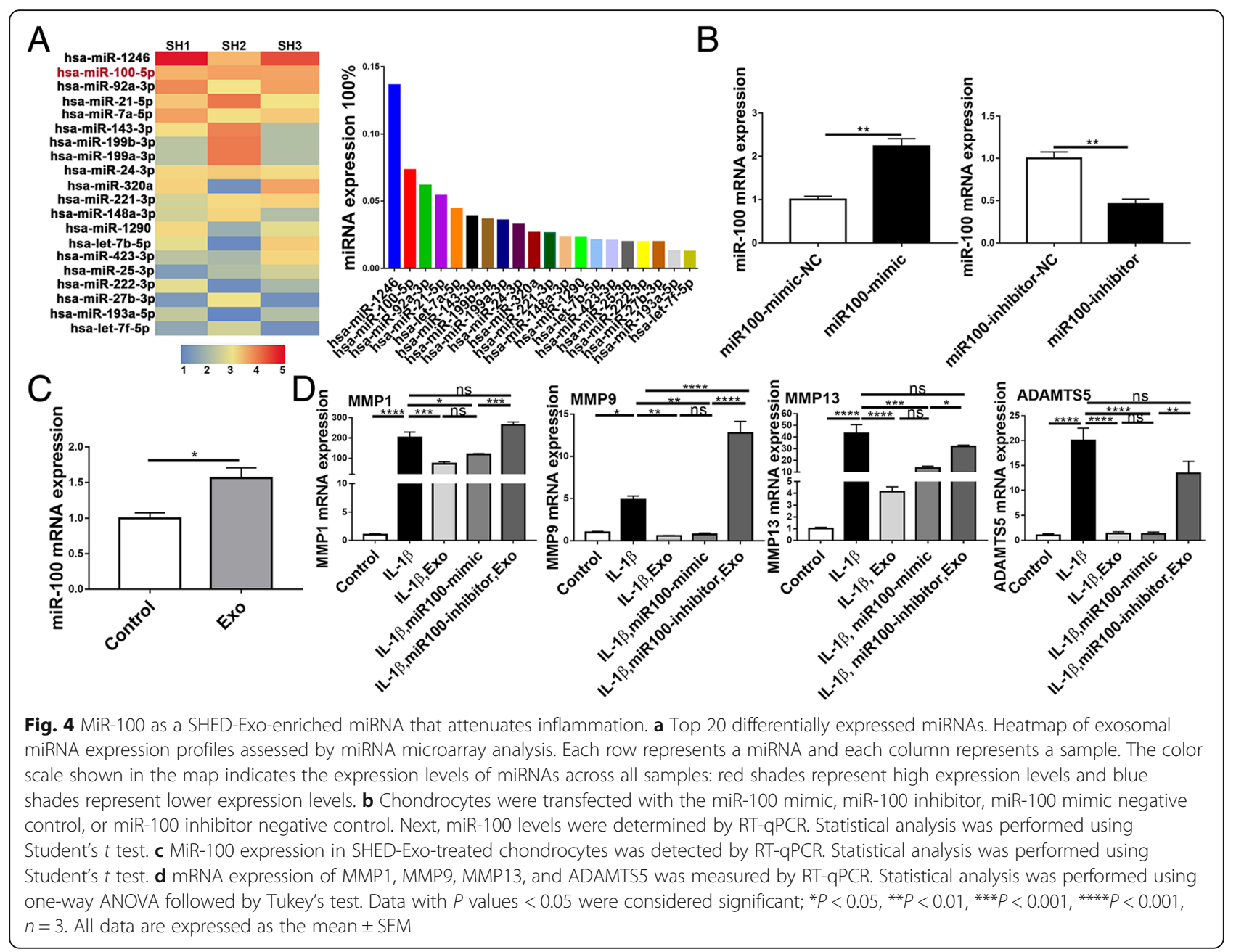

miR-100-5p from SHEDs ameliorates inflammation, miR100-5p was either overexpressed or downregulated in chondrocytes (Fig. 4b). Figure 4c shows that miR-100 was upregulated after treatment with SHED-Exos. Furthermore, the mRNA expression levels of MMP1, MMP9, MMP13, and ADAMTS5 were significantly decreased in SHEDExos-treated chondrocytes and in miR-100-overexpressing chondrocytes, whereas in the miR-100 inhibitor group, these proinflammatory enzymes were found to be upregulated (Fig. 4d).

\section{MiR-100 suppressed inflammation via repression of mammalian target of rapamycin (mTOR)}

We next clarified the molecular mechanisms underlying miR-100 regulation. The bioinformatics software TargetScan (http://www.targetscan.org) and miRanda (http://www.microrna.org) revealed that $m T O R$ mRNA contains a potential miR-100-5p-binding site.

Functional enrichment analysis revealed that the genes in the network were significantly involved in some KEGG pathways, such as the mTOR signaling pathway
(Fig. 5a). As presented in Fig. 5b-d, RT-qPCR and western blotting both revealed that mTOR was upregulated by IL- $1 \beta$ and downregulated by SHED-Exos. Overexpression of miR-100 downregulated mTOR, whereas miR-100 inhibition upregulated mTOR expression. Furthermore, to investigate the role of mTOR in TMJOA-associated inflammation, rapamycin was used to inhibit mTOR expression (Fig. 5e). As depicted in Fig. 5f, mTOR inhibition induced upregulation of miR-100. The function of mTOR was also verified as treatment with the direct inhibitor of mTOR (rapamycin) suppressed the expression of MMP1, MMP9, MMP13, and ADAMTS5 (Fig. 5g).

\section{mTOR mRNA is a direct target of miR-100}

Luciferase reporter assay results confirmed that miR100 overexpression decreased the luciferase activity in the wild-type group $(P<0.0001)$ but did not affect the mutant group $(P<0.0001$; Fig. 6a, b). Figure $6 \mathrm{c}, \mathrm{d}$ indicates that the protein level of mTOR was diminished by treatment with the miR-100 mimic, as indicated by the western blot results. 

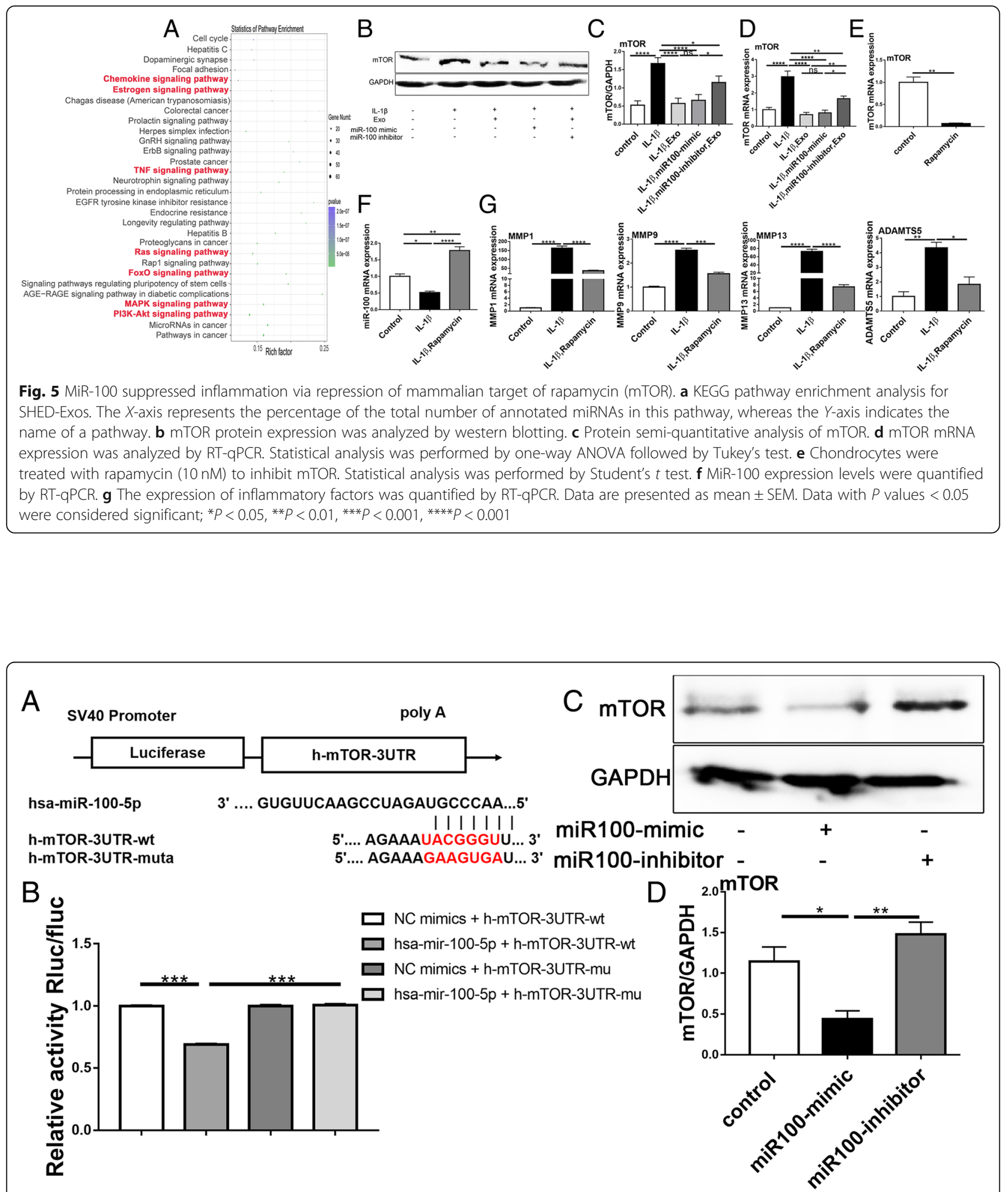

Fig. 6 mTOR mRNA is a direct target of miR-100. a Sequences of the predicted miR-100-5p target sequences in the $3^{\prime} U T R$ of $m$ TOR mRNA and its mutant containing nucleotide substitutions in the $3^{\prime} U T R$. $\mathbf{b}$ The luciferase reporter assay was conducted to test whether the $m T O R 3^{\prime} U T R$ contains a binding site for miR-100. Luciferase assays show decreased reporter activity after co-transfection of the wild-type mTOR 3'UTR plasmid with miR-100 into 293T cells. c Protein expression of mTOR visualized by western blotting. GAPDH served as an internal control. d Protein semiquantitative analysis of mTOR. Data are presented as mean $\pm \operatorname{SEM}(n=3)$. Statistical analysis was performed using one-way ANOVA followed by Tukey's test; ${ }^{*} P<0.05,{ }^{* *} P<0.01,{ }^{* * *} P<0.001,{ }^{* * *} P<0.001$ 


\section{Discussion}

This study revealed that SHED-Exos have anti-inflammatory effects in TMJOA. Furthermore, miR-100 was found to be enriched in SHED-Exos and served as an anti-inflammatory component of SHED-Exos (Fig. 7). In addition, this study demonstrated an important role of mTOR in TMJOA-associated inflammation: a downstream mediator of miR-100 action.

$\mathrm{OA}$ is the most common joint disease globally, but there is no standard treatment to prevent disease progression. Inflammation has been implicated in the pathogenesis of OA, suggesting that targeting inflammation in OA could be a promising therapeutic strategy [26]. Recently, treatment with MSC-derived exosomes has received much attention. Exosomes derived from MSCs are known play a critical part in the repair of tissue damage [27]. SHEDs, a type of MSCs, have advantages such as fewer ethical controversies, a readily accessible source, easy and minimally invasive collection, and retention of high stem cell potential such as cell proliferation, multipotency, and immunomodulatory functions compared to other MSCs [28]. Furthermore, accumulating evidence suggests that SHEDs exert curative action in various diseases including cord injury [29], bone defects [30], and ischemic brain injury [31]. Moreover, exosomes have functions similar to those of their parent cells and are safe and effective cell-free reagents. However, there was no study examining the effect of SHED-Exos on TMJOA. Based on these observations, we hypothesized that SHED-Exos may decrease the secretion of inflammatory factors in TMJOA. We were also eager to determine whether and how SHED-Exos downregulate inflammation in TMJOA.

First, to identify the presence of exosomes in a purified fraction, exosomes were confirmed by electron microscopy, NTA, and western blotting using exosome-specific biomarkers such as CD9, CD63, and TSG101 [32]. Next,

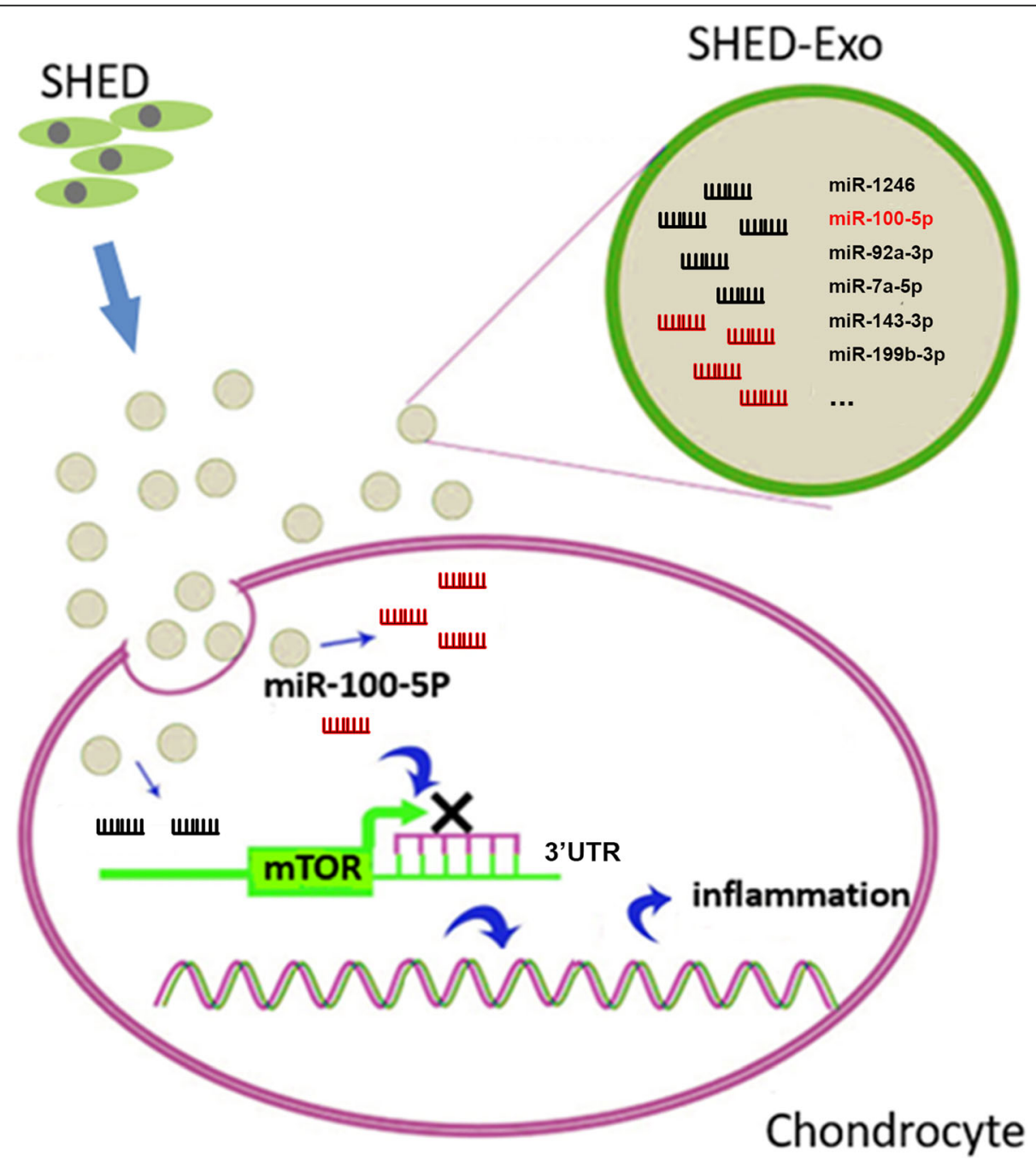

Fig. 7 SHED-Exos as an anti-inflammatory agent. SHED-EXos, enriched with miR-100-5p, as the key anti-inflammatory agent in SHED secretion through the target mTOR in chondrocytes to suppress inflammation 
to analyze the therapeutic potential of SHED-Exos in human TMJOA-associated inflammation, we conducted experiments revealing that both SHED-CM and SHEDExos have the ability to ameliorate inflammatory changes in chondrocytes; each inhibited the expression of inflammatory cytokines including IL- 6 and IL-8, and MMPs during IL-1 $\beta$-induced inflammation. Previously, administration of SHED-Exos in a rat model of traumatic brain injury was shown to downregulate inflammatory cytokines, thereby supporting the notion that SHED-Exos have anti-inflammatory functions [15]. Recently, Zhang et al. reported that MSC exosomes alleviate temporomandibular joint osteoarthritis by attenuating inflammation and restoring matrix homeostasis. However, the underlying mechanism was unclear [33].

The anti-inflammatory effects of SHED-Exos in TMJOA were detected, but the exact mechanisms behind these effects have remained unknown. MiRNAs are an important component of exosomes and have been the focus of attention for many years. Many studies suggest that the presence of miRNAs in exosomes affects a variety of diseases including OA [34]. MiRNAs bind the 3' untranslated regions (3'UTRs) of target mRNAs and suppress target protein synthesis [35]. To identify the specific SHED-derived exosomal miRNAs responsible for SHED-mediated anti-inflammatory effects, miRNA profile expression analysis was performed in the exosomes of human SHEDs. The results showed that exosomal miR-100 was significantly upregulated.

Several studies have demonstrated that miR-100 has a therapeutic potential in cancer [36]. However, the function of miRNA-100 associated with SHED-Exos in TMJOA-related inflammation is largely unknown. This study showed that an increase in miR-100 levels decreased the expression of inflammatory cytokines according to RT-qPCR. These results suggest that miR-100 plays an important role in the regulation of SHED-Exos. Moreover, Pankratz et al. recently reported that miR-100 can suppress chronic vascular inflammation, in line with our results on the effects of miR100 on TMJOA-associated inflammation [37]. The data suggest that miR-100 is required for SHED-Exos to exert anti-inflammatory effects on TMJOA.

Next, we investigated the molecular mechanisms underlying the regulation of miR-100 expression. Functional enrichment analysis revealed that PI3K/AKT, MAPK, TNF- $\alpha$, and estrogen signaling pathways are all associated with inflammation. The PI3K/AKT/mTOR pathway is believed to be related to autophagy and inflammation [38, 39]. In addition, Xue et al. have found that inhibition of PI3K/AKT/mTOR signaling attenuates the inflammatory response in rats with OA [40]. Furthermore, mTOR is closely related to proinflammatory signaling cascades of the MAPK [41], TNF- $\alpha$ [42], and estrogen pathways [43]. As the $m T O R$ gene participates in the PI3K/AKT, MAPK, TNF- $\alpha$, and estrogen signaling pathways, we focused on this gene. mTOR is the central signal integrator for nutrients and energy and performs critical functions in cell growth and division [44]. Several studies suggest that miR-100 directly targets the $m T O R$ mRNA 3'UTR and represses mTOR expression in esophageal squamous cell carcinoma and chronic vascular inflammation [37, 45]. However, the mechanism underlying the involvement of miR-100 in TMJOA-associated inflammation has not been clarified yet. Therefore, we determined whether mTOR is regulated by miR-100 in TMJOA-associated inflammation. In our study, bioinformatics analysis revealed that mTOR mRNA contains a potential miR-100-5p-binding site. Western blot and RT-qPCR results confirmed that mTOR is upregulated by IL- $1 \beta$ but is downregulated by SHED-Exos and miR-100. Conversely, mTOR was upregulated by suppression of miR-100-5p expression. In addition, rapamycin, an allosteric inhibitor of the mTOR pathway, was used to inhibit mTOR. The data showed that after rapamycin treatment, miR-100 was overexpressed and proinflammatory cytokines were downregulated [46]. Moreover, luciferase reporter assay results further confirmed that $m T O R$ mRNA is a direct target of miR-100. These data strongly indicate that the inflammation-suppressive function of miR-100 is mediated by attenuation of mTOR expression; this finding may have major implications for the mTOR pathway in TMJOAassociated inflammation. Herein, we report for the first time that SHED-Exos with miR-100-5p may participate in the maintenance of TMJ cartilage through mTOR. However, further studies are warranted to focus on the specific mechanisms that govern this process.

Further, genes in this KEGG network were significantly enriched in many other pathways, such as Ras signaling, FoxO signaling, and chemokine signaling pathways. Grabiec et al. found that reduced FoxO1 expression is required to promote the survival of fibroblast-like synoviocytes in rheumatoid arthritis [47]. Kono et al. reported that RasGRP-4, an intracellular signaling protein, is aberrantly expressed in fibroblast-like synoviocytes and helps regulate their growth; making it a candidate target for reducing proliferative synovitis and joint destruction [48]. In addition, chemokines and their receptors are found to play a significant role in arthritis processes [49]. All these pathways are related to osteoarthrosis, but the mechanisms associated with SHED need to be explored and validated further.

\section{Conclusions}

In conclusion, our results suggest that miR-100, as a SHED-Exo-enriched miRNA, suppresses inflammation via repression of mammalian target of rapamycin (mTOR). We also demonstrated the therapeutic potential of novel anti-inflammatory SHED-Exos against inflammation in 
TMJOA. As it is relatively convenient and feasible to obtain SHED-Exos, and considering their low immunogenicity, our findings may help develop a novel potential therapeutic strategy against human TMJ inflammation.

\begin{abstract}
Abbreviations
ADAMTS5: Disintegrin and metalloproteinase with thrombospondin motifs 5; CM: Conditioned medium; Exos: Exosomes; GAPDH: Glyceraldehyde 3 phosphate dehydrogenase; IL-6: Interleukin-6; IL-8: Interleukin-8; miR100: MicroRNA-100; miRNA: MicroRNA; MMP-9: Matrix metalloproteinase-9; MMP1: Matrix metalloproteinase-1; MMP13: Matrix metalloproteinase-13; MMP3: Matrix metalloproteinase-3; MSCs: Mesenchymal stem cells; mTOR: Mammalian target of rapamycin; NTA: Nanoparticle tracking analysis; OA: Osteoarthritis; RT-qPCR: Reverse transcription-quantitative polymerase chain reaction; SHEDs: Stem cells from human exfoliated deciduous teeth; TEM: Transmission electron microscopy; TMJ: Temporomandibular joint
\end{abstract}

\section{Acknowledgements}

Not applicable.

\section{Authors' contributions}

The specific contributions of the authors are as follows: (1) PL, CJ, PJ, MW and JX are responsible for the conception and design of the study; (2) PL, CJ, MW, and JX are responsible for the analysis and interpretation of the data; (3) PL and CJ are responsible for the collection and assembly of data; (4) PL and $\mathrm{CJ}$ are responsible for the drafting of the article; (5) PJ, MW, and JX are responsible for the critical revision of the article for important intellectual content; (6) PL, CJ, PJ, MW, and JX are responsible for the final approval of the article; (7) PL, CJ, PJ, MW, and JX agreed to be accountable for all aspects of the work.

\section{Funding}

This work was supported by the National Natural Science Foundation of China (grant No. 81800999) for the design of the study, the Scientific and Technological Research Program of Chongaing Municipal Education Commission (grant No. KJQN201800414) for collection, analysis, and interpretation of data, and the Program for Innovation Team Building at Institutions of Higher Education in Chongqing in 2016 (grant No. CXTDG201602006) for writing the manuscript.

\section{Availability of data and materials}

The datasets used and analyzed during the current study are available from the corresponding author on reasonable request.

\section{Ethics approval and consent to participate} Applicable.

\section{Consent for publication}

Not applicable.

\section{Competing interests}

The authors declare that they have no competing interests.

\begin{abstract}
Author details
${ }^{1}$ College of Stomatology, Chongqing Medical University, Chongqing, China. ${ }^{2}$ Chongqing Key Laboratory for Oral Diseases and Biomedical Sciences, Chongqing, China. ${ }^{3}$ Chongqing Municipal Key Laboratory of Oral Biomedical Engineering of Higher Education, Chongqing, China. ${ }^{4}$ Department of Oral and Maxillofacial Surgery, The Affiliated Hospital of Stomatology, Chongqing Medical University, No. 426, North Songshi Road, Yubei District, Chongqing 401147, China. ${ }^{5}$ Pediatric Dentistry Department, The Affiliated Hospital of Stomatology, Chongqing Medical University, No. 426, North Songshi Road, Yubei District, Chongqing 401147, China.
\end{abstract}

Received: 15 May 2019 Revised: 7 July 2019 Accepted: 14 July 2019 Published online: 29 July 2019

\section{References}

1. Zarb GA, Carlsson GE. Temporomandibular disorders: osteoarthritis. J Orofac Pain. 1999;13(4):295-306
2. Rasheed Z, Akhtar N, Haqqi TM. Advanced glycation end products induce the expression of interleukin- 6 and interleukin-8 by receptor for advanced glycation end product-mediated activation of mitogen-activated protein kinases and nuclear factor-kB in human osteoarthritis chondrocytes. Rheumatology (Oxford). 2011;50(5):838-51.

3. Deschner J, Rath-Deschner B, Agarwal S. Regulation of matrix metalloproteinase expression by dynamic tensile strain in rat fibrochondrocytes. Osteoarthr Cartil. 2006;14:264-72.

4. Martel-Pelletier J, McCollum R, Fujimoto N, Obata K, Cloutier JM. Excess of metalloproteases over tissue inhibitor of metalloprotease may contribute to cartilage degradation in osteoarthritis and rheumatoid arthritis. Lab Investig. 1994;70:807-15.

5. Wang J, Chen H, Cao P, Wu X, Zang F, Shi L, Liang L, Yuan W. Inflammatory cytokines induce caveolin-1/ $\beta$-catenin signalling in rat nucleus pulposus cell apoptosis through the p38 MAPK pathway. Cell Prolif. 2016;49:362-72.

6. Jenei-Lanzl Z, Meurer A, Zaucke F. Interleukin-1 $\beta$ signaling in osteoarthritis chondrocytes in focus. Cell Signal. 2018;53:212-23.

7. Chen K, Man C, Zhang B, Hu J, Zhu SS. Effect of in vitro chondrogenic differentiation of autologous mesenchymal stem cells on cartilage and subchondral cancellous bone repair in osteoarthritis of temporomandibular joint. Int J Oral Maxillofac Surg. 2013:42(2):240-48.

8. Miura M, Gronthos S, Zhao M, Lu B, Fisher LW, Robey PG, Shi S. SHED: stem cells from human exfoliated deciduous teeth. Proc Natl Acad Sci U S A. 2003;100(10):5807-12

9. Stanko P, Altanerova U, Jakubechova J, Repiska V, Altaner C. Dental mesenchymal stem/stromal cells and their exosomes. Stem Cells Int. 2018, 2018:1-8.

10. Yamaza T, Alatas FS, Yuniartha R, Yamaza H, Fujiyoshi JK, Yanagi Y, et al. In vivo hepatogenic capacity and therapeutic potential of stem cells from human exfoliated deciduous teeth in liver fibrosis in mice. Stem Cell Res Ther. 2015;6:171

11. Shimojima C, Takeuchi H, Jin S, Parajuli B, Hattori H, Suzumura A, et al. Conditioned medium from the stem cells of human exfoliated deciduous teeth ameliorates experimental autoimmune encephalomyelitis. J Immunol. 2016;196(10):4164-71.

12. Singla DK. Stem cells and exosomes in cardiac repair. Curr Opin Pharmacol. 2016;27:19-23.

13. Thery C, Zitvogel L, Amigorena S. Exosomes: composition, biogenesis and function. Nat Rev Immunol. 2002;2(8):569-79.

14. Whiteside TL. Exosome and mesenchymal stem cell cross-talk in the tumor microenvironment. Semin Immunol. 2018;35:69-79.

15. Johnstone RM, Adam M, Hammond JR, Orr L, Turbide C. Vesicle formation during reticulocyte maturation. Association of plasma membrane activities with released vesicles (exosomes). J Biol Chem. 1987;262(19):9412-20.

16. Bobrie A, Colombo M, Raposo G, Théry C. Exosome secretion: molecular mechanisms and roles in immune responses. Traffic. 2011;12(12):1659-68.

17. Li Y, Yang YY, Ren JL, Xu F, Chen FM, Li A. Exosomes secreted by stem cells from human exfoliated deciduous teeth contribute to functional recovery after traumatic brain injury by shifting microglia M1/M2 polarization in rats. Stem Cell Res Ther. 2017;8(1):198

18. Nishino Y, Yamada Y, Ebisawa K, Nakamura S, Okabe K, Umemura E, et al. Stem cells from human exfoliated deciduous teeth (SHED) enhance wound healing and the possibility of novel cell therapy. Cytotherapy. 2011;13(5):598-605.

19. Pivoraite U, Jarmalaviciute A, Tunaitis V, Ramanauskaite G, Vaitkuviene A, Kaseta $V$, et al. Exosomes from human dental pulp stem cells suppress carrageenaninduced acute inflammation in mice. Inflammation. 2015;38(5):1933-41.

20. Rani S, Ryan AE, Griffin MD, Ritter T. Mesenchymal stem cell-derived extracellular vesicles: toward cell-free therapeutic applications. Mol Ther. 2015;23(5):812-23.

21. Bartel DP. MicroRNAs: target recognition and regulatory functions. Cell. 2009;136(2):215-33.

22. Guo Y, Bao S, Guo W, Diao Z, Wang L, Han X, Guo W, Liu W. Bone marrow mesenchymal stem cell-derived exosomes alleviate high phosphorusinduced vascular smooth muscle cells calcification by modifying microRNA profiles. Funct Integr Genomics. 2019;19(4):633-43.

23. Thery C, Amigorena S, Raposo G, Clayton A. Isolation and characterization of exosomes from cell culture supernatants and biological fluids. Curr Protoc Cell Biol. 2006; Chapter 3:Unit 322.

24. Zhang N, Chen B, Wang W, Chen C, Kang J, Deng SQ, et al. Isolation, characterization and multi-lineage differentiation of stem cells from human exfoliated deciduous teeth. Mol Med Rep. 2016;14:95-102. 
25. Narayanan R, Huang CC, Ravindran S. Hijacking the cellular mail: exosome mediated differentiation of mesenchymal stem cells. Stem Cells Int. 2016; 2016:3808674

26. Scanzello CR, McKeon B, Swaim BH, DiCarlo E, Asomugha EU, Kanda V, et al. Synovial inflammation in patients undergoing arthroscopic meniscectomy: molecular characterization and relationship to symptoms. Arthritis Rheum. 2011;63(2):391-400.

27. Lai RC, Arslan F, Lee MM, Sze NS, Choo A, Chen TS, et al. Exosome secreted by MSC reduces myocardial ischemia/reperfusion injury. Stem Cell Res. 2010;4(3):214-22.

28. Yamaza T, Kentaro A, Chen C, Liu Y, Shi Y, Gronthos S, Wang S, Shi S. Immunomodulatory properties of stem cells from human exfoliated deciduous teeth. Stem Cell Res Ther. 2010;1(1):5.

29. Sakai K, Yamamoto A, Matsubara K, Nakamura S, Naruse M, Yamagata M, Sakamoto K, Tauchi R, Wakao N, Imagama S, Hibi H, Kadomatsu K, Ishiguro N, Ueda M. Human dental pulpderived stem cells promote locomotor recovery after complete transection of the rat spinal cord by multiple neuro-regenerative mechanisms. J Clin Invest. 2012;122(1):80-90.

30. Seo BM, Sonoyama W, Yamaza T, Coppe C, Kikuiri T, Akiyama K, et al. SHED repair critical-size calvarial defects in mice. Oral Dis. 2008;14(5):428-34.

31. Inoue $T$, Sugiyama $M$, Hattori $H$, Wakita $H$, Wakabayashi $T$, Ueda M. Stem cells from human exfoliated deciduous tooth-derived conditioned medium enhance recovery of focal cerebral ischemia in rats. Tissue Eng Part A. 2013; 19:24-9.

32. Bobrie A, Colombo M, Krumeich S, Raposo G, Théry C. Diverse subpopulations of vesicles secreted by different intracellular mechanisms are present in exosome preparations obtained by differential ultracentrifugation. J Extracell Vesicles. 2012;1(1):18397.

33. Zhang S, Teo KYW, Chuah SJ, Lai RC, Lim SK, Toh WS. MSC exosomes alleviate temporomandibular joint osteoarthritis by attenuating inflammation and restoring matrix homeostasis. Biomaterials. 2019;200:35-47.

34. Meng F, Zhang Z, Chen W, Huang G, He A, Hou C, et al. MicroRNA-320 regulates matrix metalloproteinase-13 expression in chondrogenesis and interleukin-1 beta-induced chondrocyte responses. Osteoarthr Cartil. 2016;24: 932-41

35. Didiano D, Hobert O. Molecular architecture of a miRNA-regulated 3' UTR. RNA. 2008;14(7):1297-317.

36. Xu C, Zeng Q, Xu W, Jiao L, Chen Y, Zhang Z, et al. miRNA-100 inhibits human bladder urothelial carcinogenesis by directly targeting mTOR. Mol Cancer Ther. 2013;12(2):207-19.

37. Pankratz F, Hohnloser C, Bemtgen X, Jaenich C, Kreuzaler S, Hoefer I, et al. MicroRNA-100 suppresses chronic vascular inflammation by stimulation of endothelial autophagy. Circ Res. 2018;122(3):417-32.

38. Chang Y, Kong R. Ganoderic acid A alleviates hypoxia-induced apoptosis, autophagy, and inflammation in rat neural stem cells through the PI3KVAKT/ mTOR pathways. Phytother Res. 2019;33(5):1448-56.

39. Tong XP, Chen Y, Zhang SY, Xie T, Tian M, Guo MR, et al. Key autophagic targets and relevant small-molecule compounds in cancer therapy. Cell Prolif. 2015:48(1):7-16.

40. Xue JF, Shi ZM, Zou J, Li XL. Inhibition of PI3K/AKT/mTOR signaling pathway promotes autophagy of articular chondrocytes and attenuates inflammatory response in rats with osteoarthritis. Biomed Pharmacother. 2017:89:1252-61.

41. Pernice Helena F, Rico S, Kiebler Michael A, et al. mTOR and MAPK: from localized translation control to epilepsy. BMC Neurosci. 2016;17:73.

42. Wang $C, H u$ L, Zhao L, Yang $P$, Moorhead JF, Varghese $Z$, et al. Inflammatory stress increases hepatic CD36 translational efficiency via activation of the mTOR signalling pathway. PLoS One. 2014;9(7):e103071.

43. Pratap UP, Sharma HR, Mohanty A, Kale P, Gopinath S, Hima L, et al. Estrogen upregulates inflammatory signals through $\mathrm{NF}-\mathrm{KB}$, IFN- $\gamma$, and nitric oxide via Akt/mTOR pathway in the lymph node lymphocytes of middleaged female rats. Int Immunopharmacol. 2015;29(2):591-98

44. Zoncu R, Efeyan A, Sabatini DM. mTOR: from growth signal integration to cancer, diabetes and 516 ageing. Nat Rev Mol Cell Biol. 2011;12:21-35.

45. Zhang $N$, Fu H, Song L, Ding $Y$, Wang $X$, Zhao C, Zhao $Y$, Jiao F, Zhao $Y$. MicroRNA-100 promotes migration and invasion through mammalian target of rapamycin in esophageal squamous cell carcinoma. Oncol Rep. 2014;32: 1409-18.

46. Bonegio RG, Fuhro R, Wang Z, Valeri CR, Andry C, Salant DJ, Lieberthal W. Rapamycin ameliorates proteinuria-associated tubulointerstitial inflammation and fibrosis in experimental membranous nephropathy. J Am Soc Nephrol. 2005;16(7):2063-72.
47. Grabiec AM, Angiolilli C, Hartkamp LM, van Baarsen LG, Tak PP, Reedquist KA. JNK-dependent downregulation of FoxO1 is required to promote the survival of fibroblast-like synoviocytes in rheumatoid arthritis. Ann Rheum Dis. 2015;74:1763-71.

48. Kono M, Yasuda S, Stevens RL, Koide H, Kurita T, Shimizu Y, et al. Ras guanine nucleotide-releasing protein 4 is aberrantly expressed in the fibroblast-like synoviocytes of patients with rheumatoid arthritis and controls their proliferation. Arthritis Rheumatol. 2015;67:396-407.

49. Sato H, Muraoka S, Kusunoki N, Masuoka S, Yamada S, Ogasawara H, et al. Resistin upregulates chemokine production by fibroblast-like synoviocytes from patients with rheumatoid arthritis. Arthritis Res Ther. 2017;19:263.

\section{Publisher's Note}

Springer Nature remains neutral with regard to jurisdictional claims in published maps and institutional affiliations.
Ready to submit your research? Choose BMC and benefit from:

- fast, convenient online submission

- thorough peer review by experienced researchers in your field

- rapid publication on acceptance

- support for research data, including large and complex data types

- gold Open Access which fosters wider collaboration and increased citations

- maximum visibility for your research: over $100 \mathrm{M}$ website views per year

At BMC, research is always in progress.

Learn more biomedcentral.com/submissions 